\title{
Article \\ C-Arm Cone-Beam CT Virtual Navigation versus Conventional CT Guidance in the Transthoracic Lung Biopsy: A Case-Control Study
}

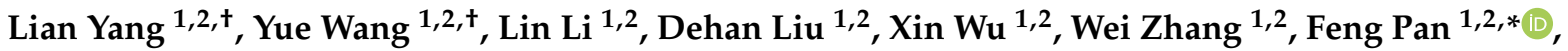 \\ Huimin Liang ${ }^{1,2}$ and Chuansheng Zheng ${ }^{1,2}$
}

1 Department of Radiology, Union Hospital, Tongji Medical College, Huazhong University of Science and Technology, Jiefang Avenue \#1277, Wuhan 430022, China; yanglian@hust.edu.cn (L.Y.); m202076089@hust.edu.cn (Y.W.); 2015xh0933@hust.edu.cn (L.L.); 2015xh0934@hust.edu.cn (D.L.); 2009xh1135@hust.edu.cn (X.W.); 2009xh1108@hust.edu.cn (W.Z.); 1988xh0737@hust.edu.cn (H.L.); cszheng@hust.edu.cn (C.Z.)

2 Hubei Province Key Laboratory of Molecular Imaging, Jiefang Avenue \#1277, Wuhan 430022, China

* Correspondence: uh_fengpan@hust.edu.cn

+ These authors contributed equally to this work.

check for

updates

Citation: Yang, L.; Wang, Y.; Li, L.; Liu, D.; Wu, X.; Zhang, W.; Pan, F.; Liang, H.; Zheng, C. C-Arm Cone-Beam CT Virtual Navigation versus Conventional CT Guidance in the Transthoracic Lung Biopsy: A Case-Control Study. Diagnostics 2022, 12, 115. https://doi.org/10.3390/ diagnostics12010115

Academic Editor: Wonjun Ji

Received: 21 November 2021

Accepted: 2 January 2022

Published: 5 January 2022

Publisher's Note: MDPI stays neutral with regard to jurisdictional claims in published maps and institutional affiliations.

Copyright: (C) 2022 by the authors. Licensee MDPI, Basel, Switzerland. This article is an open access article distributed under the terms and conditions of the Creative Commons Attribution (CC BY) license (https:// creativecommons.org/licenses/by/ $4.0 /)$.

\begin{abstract}
C-arm cone-beam computed tomography (CBCT) virtual navigation-guided lung biopsy has been developed in the last decade as an alternative to conventional CT-guided lung biopsy. This study aims to compare the biopsy accuracy and safety between these two techniques and explores the risk factors of biopsy-related complications. A total of 217 consecutive patients undergoing conventional CT- or C-arm CBCT virtual navigation-guided lung biopsy from 1 June 2018 to 31 December 2019 in this single-center were retrospectively reviewed. Multiple factors (e.g., prior emphysema, lesion size, etc.) were compared between two biopsy techniques. The risk factors of complications were explored by using logistic regression. The patients' median age and male-to-female ratio were 63 years and 2.1:1, respectively. Eighty-two (82) patients (37.8\%) underwent conventional CT-guided biopsies, and the other 135 patients (62.2\%) C-arm CBCT virtual navigation-guided biopsies. Compared with patients undergoing C-arm CBCT virtual navigation-guided lung biopsies, patients undergoing conventional CT-guided lung biopsies showed higher needle repositioning rate, longer operation time, and higher effective dose of X-ray (52.4\% vs. 6.7\%, $25 \mathrm{~min}$ vs. $15 \mathrm{~min}$, and $13.4 \mathrm{mSv}$ vs. $7.6 \mathrm{mSv}$, respectively; $p<0.001$, each). In total, the accurate biopsy was achieved in 215 of 217 patients $(99.1 \%)$, without a significant difference between the two biopsy techniques $(p=1.000)$. The overall complication rates, including pneumothorax and pulmonary hemorrhage/hemoptysis, are $26.3 \%$ (57/217), with most minor complications $(56 / 57,98.2 \%)$. The needle repositioning was the only independent risk factor of complications with an odds ratio of $6.169(p<0.001)$. In conclusion, the C-arm CBCT virtual navigation is better in percutaneous lung biopsy than conventional CT guidance, facilitating needle positioning and reducing radiation exposure. Needle repositioning should be avoided because it brings about more biopsy-related complications.
\end{abstract}

Keywords: lung neoplasms; image-guided biopsy; cone-beam computed tomography; postoperative complications; risk factors

\section{Introduction}

The conventional CT-guided lung biopsy is one of the pathologically diagnostic methods for solid pulmonary neoplasms, with a diagnostic sensitivity for malignancies of more than $90 \%$ [1,2]. It is minimally invasive with excellent safety and can be performed under local anesthesia [2]. It is specifically indicated for peripheral lung lesions that bronchoscopy cannot reach $[2,3]$.

Nevertheless, the technique of conventional CT-guided lung biopsy has some shortcomings. First, there is a lack of real-time monitoring during the puncture process [4,5]. The 
operator can only advance the needle based on the preset route from the initial CT scan $[4,5]$. If the patient's respiration is not cooperative, the needle may miss the target lesion during the puncture, and the process must be repeated. As a result, it probably increases the iatrogenic injury to the lung parenchyma, prolongs the procedure time, and enhances the radiation exposure because of numerous CT scans. Second, the needle placement is limited by the axial imaging plane of the CT scanner [6]. Thus, it is difficult to achieve an ideal puncture path setting under conventional CT guidance in some cases.

Since a decade ago, C-arm cone-beam CT (CBCT) virtual navigation-guided lung biopsy has been developed due to its high technical success rate $(>99 \%)$, high accuracy $(>95 \%)$, and good safety [5,7-14]. The C-arm CBCT system consists of flat-panel fluoroscopy and CBCT scanner. It can use 3-dimensional (3D) reconstruction of CBCT imaging to achieve virtual navigation and monitor the needle puncture under fluoroscopy $[7,9,10,12-14]$. The real-time fluoroscopy can give direct feedback of the opacified solid lesion movement due to respiration, facilitating precise respiratory control under the virtual navigation overlay $[7,10,14]$. Besides this, due to the free 3D rotation of the C-arm, C-arm CBCT can realize free-angle puncture navigation, recognizing the best-preset puncture path [7-13]. However, there has been a minimal number of studies comprehensively comparing the conventional CT- and C-arm CBCT virtual navigation-guided lung biopsies, especially for the lung lesions firstly indicated for transthoracic biopsies [2,3]. Therefore, our study intends to compare the diagnostic accuracy and safety between these techniques and explore the risk factors of biopsy-related complications.

\section{Methods}

\subsection{Patients}

The consecutive electronic hospitalization records from 1 June 2018 to 31 December 2019 were retrospectively reviewed in this single center (Western Campus affiliated to Union Hospital, Wuhan, China). A total of 217 patients undergoing conventional CTor C-arm CBCT virtual navigation-guided lung biopsy for the solid pulmonary lesions were included. The clinical data, including procedure records and pathological results, were collected.

\subsection{Pre-Procedure Evaluation}

We followed the expert recommendations and international guidelines in lung biopsy evaluation, planning, and performance [2,3]. Before the biopsy, enhanced chest CT and laboratory investigations (e.g., blood routine, coagulation test, etc.) were routinely performed. Further positron emission tomography was carried out if it was hard to differentiate between the neoplasm and post-obstructive atelectasis. After completing the preoperative examinations, a multidisciplinary team (incl. interventionalist, thoracic surgeon, pulmonologist, and anesthetist) evaluated and decided whether the lung biopsy needed to be executed. If lung biopsy was indicated, transthoracic lung biopsy was considered in case of a non-candidate for bronchoscopy, an inaccessible pulmonary lesion by bronchoscopy, a prior non-diagnostic bronchoscopic biopsy, or a subjective requirement by the patient [2]. The choice of $\mathrm{CT}$ or $\mathrm{CBCT}$ guidance depended on the availability of equipment in the schedule. Before the biopsy, the operators should review the prior enhanced chest $\mathrm{CT}$ and other relevant imaging to clarify the target required to be biopsied. In the planning of needle puncture, structures including ribs, vascular vessels, blebs, bullae, central bronchi, and fissures should be dodged [2]. In addition, if a lung lesion demonstrated central cavity or necrosis, the periphery was targeted. The written informed consent from the patient was acquired $24 \mathrm{~h}$ before the procedure. All procedures were performed by the same team (HL, FP, LL, and DL) under the supervision of one senior interventionalist (HL with 25 years and one year of experience in CT- and C-arm CBCT virtual navigation-guided biopsy before, respectively). 


\subsection{Conventional CT Guidance in Lung Biopsy}

A 64-slice CT scanner (Philips Ingenuity Core128, Philips Medical Systems, Best, The Netherland) was used. The image acquisition parameters included: $120 \mathrm{kVp}$ with adaptive current modulation, a pitch of 0.999 , collimation of $64 \times 0.625 \mathrm{~mm}$, a gantry rotation time of $0.5 \mathrm{~s}$, and a DoseRight index of 18. Axial CT images were reconstructed (slice thickness of $1.5 \mathrm{~mm}$ and increment of $1.5 \mathrm{~mm}$ ) with iterative reconstruction (iDose level 5, Philips Healthcare). An initial scan was performed to establish the eligible puncture route to the target lesion under breath-holding after a full aspiration. After locating the target slice, the skin entry point was determined using the cross of a radiopaque grid placed on the patient skin and the perpendicular laser beam (Figure 1a) [2]. After dermal sterilization, draping, and local anesthesia, the patient was asked to hold the breath again, and a $17 \mathrm{G}$ coaxial needle (Bard ${ }^{\circledR}$ TruGuide $^{\circledR}, 17 \mathrm{G}^{*} 13.8 \mathrm{~cm}$, Bard Care, Covington, OH, USA) was advanced toward the target following the preset route and depth. Afterward, the chest CT was re-performed to check whether an eligible needle position was reached (Figure 1b). If not, the needle repositioning was carried out until the needle tip reached the target.

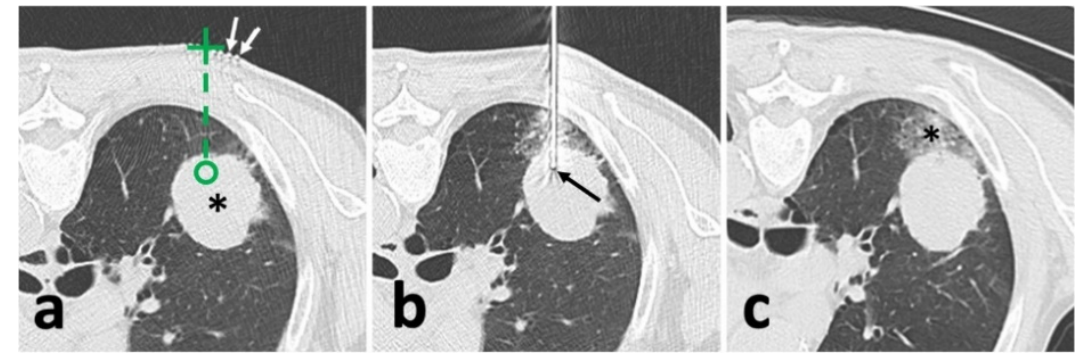

Figure 1. Illustration of conventional CT-guided biopsy. (a), localization with opaque grid marking (white arrow) and needle route planning (green dash line) targeting the solid lesion $\left({ }^{*}\right)$; (b), after needle puncture, CT showed an appropriate location of the needle tip (black arrow) within the solid lesion; (c), after removal of the needle and biopsy instrument, post-biopsy CT demonstrated pulmonary hemorrhage ${ }^{*}$ ) alongside the puncture route without the findings of pneumothorax.

\subsection{CBCT Guidance in Lung Biopsy}

A rotating angiography system (Artis Zee, Siemens Healthcare, Erlangen, Germany) was used to obtain 3D chest CBCT images during the procedure. Similarly, the patient was asked to hold a breath after a full aspiration during the initial image acquisition. A $6 \mathrm{~s}$ rotational scan generated 397 projection images with an angular step of $0.5^{\circ}$ and a pulse length of $3.2 \mathrm{~ms}$. A tube voltage of $90 \mathrm{kV}$ with a current of $273.5 \mathrm{~mA}$ was set up. Then, the projection images were automatically transmitted to a post-processing workstation (Syngo X Workplace, Siemens Healthcare) for multi-planar reconstruction (MPR) of axial, sagittal, and coronal orientations and 3D volume reconstruction. In the next step, the needle path was set up using a commercial plug-in (Syngo iGuide, Siemens Healthcare) $[15,16]$. After manually selecting the skin entry point and target lesion position in MPR (Figure 2a), a virtual path with displayed angulation and length was generated and overlayed to realtime fluoroscopy to navigate the puncture process. Then, the operator rotated the C-arm to the Bull's Eye View and turned on the laser navigation system on the flat panel to locate the skin entry point (Figure 2b). After dermal sterilization, draping, and local anesthesia, the patient was asked to hold a breath again. Then, under real-time fluoroscopy monitoring, a $17 \mathrm{G}$ coaxial needle was advanced along the virtual path until the planned target position was reached (Figure 2c). Afterward, a CBCT scan was re-performed to identify whether the needle repositioning was needed (Figure $2 \mathrm{~d}$ ). 

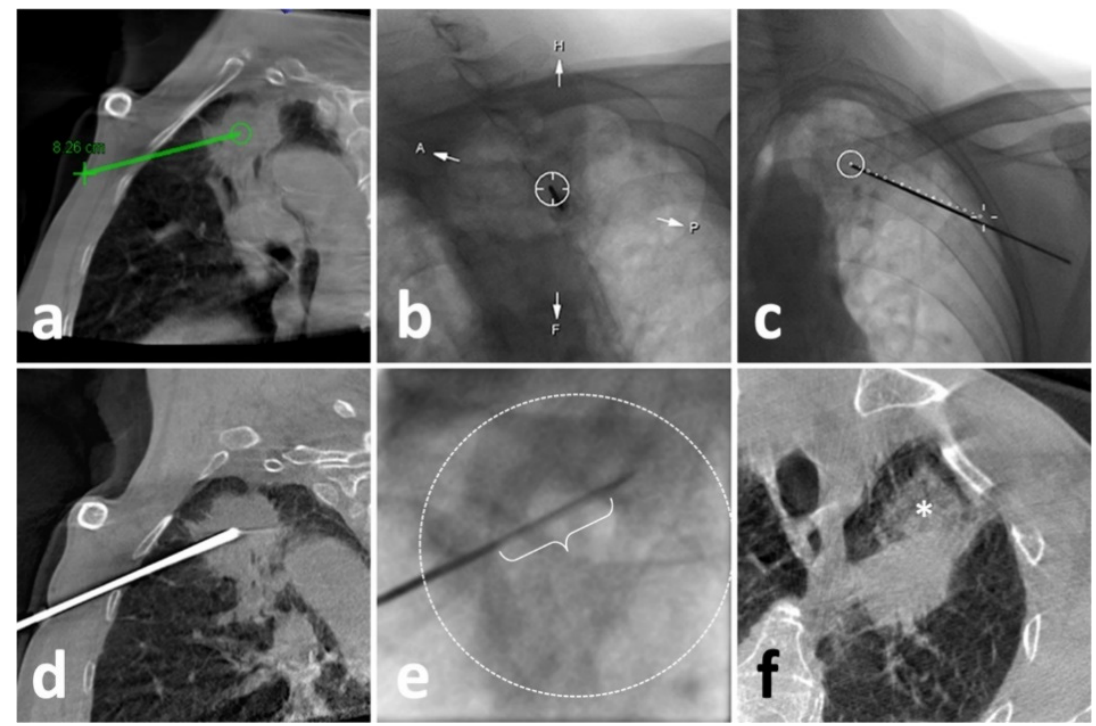

Figure 2. Illustration of C-arm CBCT virtual navigation-guided biopsy. (a), localization of the lesion (solid mass at left upper lobe) and needle route planning (green line); (b), fluoroscopy-guided puncture under virtual navigation at Bull's Eye View Position; the middle circle indicates the target which was accurately punctured by a 17-G needle; (c), Fluoroscopic check of the needle, which matched perfectly with the virtual path (dash line); (d), a re-examination of CBCT showed eligible needle tip within the solid lesion; (e), the performance of biopsy under real-time fluoroscopy, in which the sampling part of the biopsy instrument (curly bracket) showed appropriate location within the opacified lesion (dash circle); (f), the post-biopsy CBCT demonstrated pulmonary hemorrhage ${ }^{*}$ ) alongside the puncture route without the findings of pneumothorax.

\subsection{Lung Biopsy}

After achieving an ideal position of the coaxial needle, the stylet was removed. Then, a biopsy instrument (Bard ${ }^{\circledR}$ Max-Core ${ }^{\circledR}$ Disposable Core Biopsy Instrument, $18 \mathrm{G}^{*} 16 \mathrm{~cm}$, Bard Care, Covington, OH, USA) was advanced to obtain samples (so-called "coaxial cutting needle technique") (Figure 2e) $[10,17,18]$. Two to eight samples were obtained based on the pathological and other genetic test demands. Afterward, the needle was removed, and the post-biopsy CT or CBCT was routinely acquired to identify biopsyrelated complications (Figures $1 \mathrm{c}$ and $2 \mathrm{f}$ ).

\subsection{Study Goals}

We compared prior emphysema history, lesion size, technical success, needle repositioning, operation time, an effective X-ray dose, complications, and biopsy accuracy between patients undergoing conventional CT- and C-arm CBCT virtual navigation-guided lung biopsies. The lesion size was defined as an average of long- and short-axis measurements in the maximum lesion section of axial CT images following the radiological statement from the Fleischner Society (Figure 3a) [19]. Technical success was defined as satisfactory biopsy materials that qualified for pathologic analysis [20]. The needle repositioning was defined as the pull-back adjustment or re-puncture when the needle tip did not reach the planning target $[5,6]$. During the procedure, operation time was recorded from the start of the initial CT/CBCT scan to the end of the post-biopsy CT/CBCT scan. The effective dose of conventional $\mathrm{CT}$ was calculated by using a dose-linear product $(\mathrm{DLP})^{*} \mathrm{~K}$-factor and fluoroscopy /CBCT by using a dose-area product (DAP)*dose conversion coefficient [21]. For conventional chest CT, $\mathrm{k}$-factor was $0.0146 \mathrm{mSv} / \mathrm{mGy}{ }^{*} \mathrm{~cm}$ [22] For chest fluoroscopy/CBCT of the Artis system, the dose conversion coefficient was $0.0017 \mathrm{mSv} / \mu \mathrm{Gy}^{*} \mathrm{~m}^{2}[10,14]$. The accurate biopsy was defined as the biopsy pathological result that was: 1 . confirmed by the following surgery; or 2 . supported by subsequent 
clinical course for at least one year (e.g., growth or metastasis of malignancies, stable or regression of benign lesions, etc.) $[6,7,10,23]$.
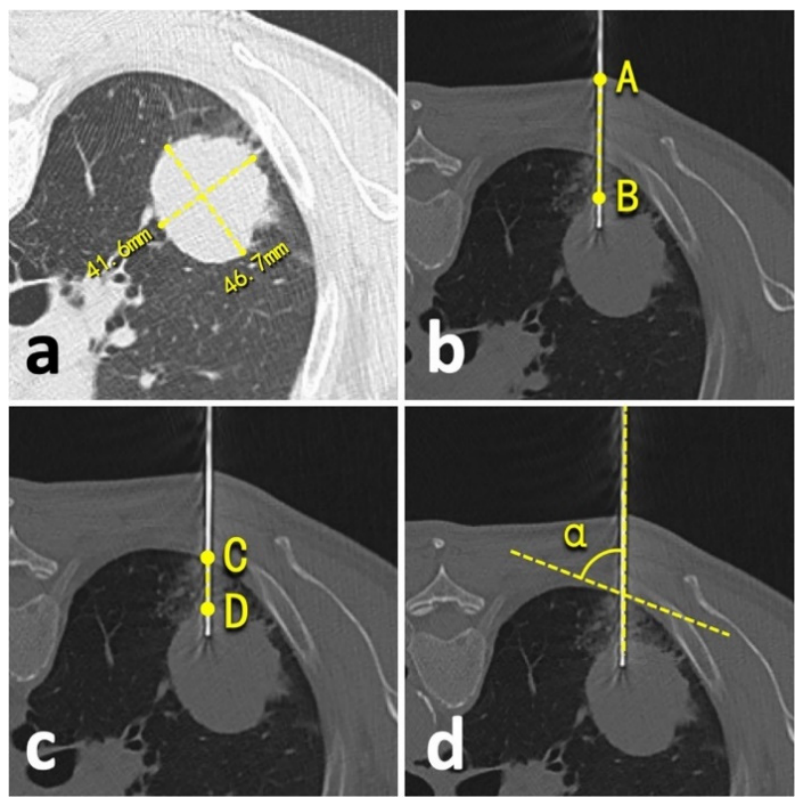

Figure 3. Illustration of quantitative measurements. (a), lesion size; (b), lesion-skin distance; (c), lesionpleural distance; (d), needle-pleural angle.

The biopsy-related complications were collected and classified from Grade- $A$ to $-E$ under the standards of the Society of Interventional Radiology [24]. A minor complication was defined as Grade- $A$ or $-B$ complication, and a major complication as Grade- $C$ to $-E$ complication [24]. Besides this, the risk factors of complication were comprehensively explored, including prior emphysema history, patient position during the biopsy, lesion size, needle repositioning, lesion-skin distance along the needle path (from lesion border to skin) (Figure 3b), lesion-pleural distance along the needle path (from lesion border to pleura) (Figure 3c), needle-pleural angle (Figure 3d), and the number of obtained samples $[4,5,19,20,23]$. Two independent radiologists performed all measurements (LY and YW, with 23- and 2-year experience in thoracic radiology, respectively), and the average of the measurements was involved in the final analysis.

\subsection{Statistical Analysis}

Statistical analyses were performed using SPSS Statistics Software (version 26; IBM, New York, NY, USA). Quantitative data were presented as median with inter-quantile range (IQR), while the counting data were presented as count with the percentage of the total. The quantitative data comparisons were evaluated using Mann-Whitney tests, according to the non-normal distribution assessed by Shapiro-Wilk tests. Chi-square tests were performed to assess categorical variables between different groups, and Fisher's exact test was implemented instead of the Chi-square test if the expected count was less than five. Univariate and multivariate logistic regression tests using forward conditional methods were performed to investigate the independent risk factors for biopsy-related complications. Odds ratio (OR) with 95\% confidence interval $(95 \% \mathrm{CI})$ was calculated. Inter-observer agreement of the measurements was assessed using intraclass correlation efficient (ICC) analysis by applying a two-way random model. All tests were two-sided, and a $p$-value of $<0.01$ was defined as a statistical significance. 


\section{Results}

\subsection{Patient Characteristics}

The patients' median age and male-to-female ratio were 63 years (IQR: 54-69 years) and 2.1:1, respectively. More than one-fifth of patients $(60 / 217,27.6 \%)$ had prior emphysema history. Among them, 82 patients $(82 / 217,37.8 \%)$ underwent conventional CT-guided lung biopsies, while the other 135 patients $(135 / 217,62.2 \%)$ underwent C-arm CBCT virtual navigation-guided lung biopsies. The biopsied lesions were mainly located in the right and left upper lung lobes (31.8\% and $28.6 \%$, respectively). The median lesion size was $40.0 \mathrm{~mm}$ (IQR: 26.8-54.8 mm). More details are summarized in Table 1.

Table 1. Basic characteristics.

\begin{tabular}{|c|c|}
\hline & Results, $\mathrm{n}=217$ \\
\hline \multicolumn{2}{|l|}{ Sex } \\
\hline Male & $146(67.3 \%)$ \\
\hline Female & $71(32.7 \%)$ \\
\hline Age (years) (IQR) & $63(54-69)$ \\
\hline Prior emphysema history & $60(27.6 \%)$ \\
\hline Pleural effusion & $2(0.9 \%)$ \\
\hline \multicolumn{2}{|l|}{ Lung lesion location ${ }^{1}$} \\
\hline Left upper lobe & $62(28.6 \%)$ \\
\hline Apicoposterior segment (B1/2) & $30(13.8 \%)$ \\
\hline Anterior segment (B3) & $13(6.0 \%)$ \\
\hline Superior lingular segment (B4) & $11(5.1 \%)$ \\
\hline Inferior lingular segment (B5) & $8(3.7 \%)$ \\
\hline Left lower lobe & $32(14.7 \%)$ \\
\hline Superior segment (B6) & $8(3.7 \%)$ \\
\hline Anteromedial segment (B7/8) & $9(4.1 \%)$ \\
\hline Lateral segment (B9) & $6(2.8 \%)$ \\
\hline Posterior segment (B10) & $9(4.1 \%)$ \\
\hline Right upper lobe & $69(31.8 \%)$ \\
\hline Apical segment (B1) & $32(14.7 \%)$ \\
\hline Posterior segment (B2) & $24(11.1 \%)$ \\
\hline Anterior segment (B3) & $13(6.0 \%)$ \\
\hline Right middle lobe & $10(4.6 \%)$ \\
\hline Lateral segment (B4) & $8(3.7 \%)$ \\
\hline Medial segment (B5) & $2(0.9 \%)$ \\
\hline Right lower lobe & $44(20.3 \%)$ \\
\hline Superior segment (B6) & $13(6.0 \%)$ \\
\hline Medial segment (B7) & $3(1.4 \%)$ \\
\hline Anterior segment (B8) & $11(5.1 \%)$ \\
\hline Lateral segment (B9) & $8(3.7 \%)$ \\
\hline Posterior segment (B10) & $9(4.1 \%)$ \\
\hline Lesion size $(\mathrm{mm})^{2}$ & $40.0(26.8-54.8)$ \\
\hline \multirow{3}{*}{$\begin{array}{c}\text { Biopsy techniques } \\
\text { Conventional CT-guided lung biopsy } \\
\text { C-arm CBCT virtual navigation-guided lung } \\
\text { biopsy }\end{array}$} & \\
\hline & $82(37.8 \%)$ \\
\hline & $135(62.2 \%)$ \\
\hline
\end{tabular}

\subsection{Comparisons between Conventional CT- and C-arm CBCT Virtual Navigation-Guided Lung Biopsies}

The technical success of the biopsy was achieved in all patients $(217 / 217,100.0 \%)$. Compared with patients undergoing C-arm CBCT virtual navigation-guided lung biopsies, patients undergoing conventional CT-guided lung biopsies showed higher needle repositioning incidence, longer operation time, a higher effective dose of X-ray, and less obtained samples (52.4\% vs. 6.7\%, $25 \mathrm{~min}$ vs. $15 \mathrm{~min}, 13.4 \mathrm{mSv}$ vs. $7.6 \mathrm{mSv}$, and four vs. 5, 
respectively; $p<0.001$, each) (Table 2). On the other hand, our results showed no significant differences in age, sex, lesion size, lesion-skin/lesion-pleural distance along the needle path, needle-pleural angle, complications, and biopsy accuracy between the two biopsy groups (Table 2). Overall, the accurate biopsy was achieved in 215 of 217 patients (99.1\%) (Table 2). Two (2) patients $(2 / 217,0.9 \%)$ diagnosed with inflammation by biopsies were finally confirmed with malignancies after surgical resection (one was small cell carcinoma; the other was the metastasis of clear cell renal cell carcinoma) (Table 2).

Table 2. Comparisons between conventional CT- and C-arm CBCT virtual navigation-guided lung biopsies.

\begin{tabular}{|c|c|c|c|c|}
\hline & Total, $\mathrm{n}=217$ & $\begin{array}{l}\text { Conventional CT-Guided } \\
\text { Lung Biopsy, } n=82 \text {. }\end{array}$ & $\begin{array}{l}\text { C-Arm CBCT Virtual } \\
\text { Navigation-Guided Lung } \\
\text { Biopsy, } n=135 \text {. }\end{array}$ & $p$-Value ${ }^{1}$ \\
\hline Age (years) & $63(54-69)$ & $62(54-70)$ & $63(53-68)$ & 1.000 \\
\hline$\leq 63$ & $115(53.0 \%)$ & $47(57.3 \%)$ & $68(50.4 \%)$ & \multirow{3}{*}{0.320} \\
\hline$>63$ & $102(47.0 \%)$ & $35(42.7 \%)$ & $67(49.6 \%)$ & \\
\hline Sex & & & & \\
\hline Male & $146(67.3 \%)$ & $53(64.6 \%)$ & $93(68.9 \%)$ & \multirow[b]{2}{*}{0.517} \\
\hline Female & $71(32.7 \%)$ & $29(35.4 \%)$ & $42(31.1 \%)$ & \\
\hline Prior emphysema history & $60(27.6 \%)$ & $16(19.5 \%)$ & $44(32.6 \%)$ & 0.037 \\
\hline Lesion size $(\mathrm{mm})^{2,3}$ & $40.0(26.8-54.8)^{4}$ & $42.3(30.3-54.8)$ & $36.8(23.8-54.8)$ & 0.250 \\
\hline$<40.0$ & $110(50.7 \%)$ & $36(43.9 \%)$ & $74(54.8 \%)$ & \multirow{2}{*}{0.119} \\
\hline$>40.0$ & $107(49.3 \%)$ & $46(56.1 \%)$ & $61(45.2 \%)$ & \\
\hline Technical success & $217(100.0 \%)$ & $82(100.0 \%)$ & $135(100.0 \%)$ & 1.000 \\
\hline \multicolumn{5}{|l|}{ Position } \\
\hline Supine & $113(52.1 \%)$ & $39(47.6 \%)$ & $74(54.8 \%)$ & \multirow{3}{*}{0.396} \\
\hline Prone & $99(45.6 \%)$ & $40(48.8 \%)$ & $59(43.7 \%)$ & \\
\hline Lateral recumbent & $5(2.3 \%)$ & $3(3.7 \%)$ & $2(1.5 \%)$ & \\
\hline Number of needle repositioning & $0(0-0)$ & $1(0-2)$ & $0(0-0)$ & $<0.001$ \\
\hline Needle repositioning & $52(24.0 \%)$ & $43(52.4 \%)$ & $9(6.7 \%)$ & $<0.001$ \\
\hline $\begin{array}{l}\text { Lesion-skin distance along the } \\
\text { needle path }(\mathrm{mm})^{3}\end{array}$ & $51.7(37.9-67.5)^{5}$ & $51.3(38.2-64.5)$ & $51.8(35.1-68.4)$ & 0.865 \\
\hline$\leq 51.7$ & $108(49.8 \%)$ & $41(50.0 \%)$ & $67(49.6 \%)$ & \multirow{2}{*}{0.958} \\
\hline$>51.7$ & $109(50.2 \%)$ & $41(50.0 \%)$ & $68(50.4 \%)$ & \\
\hline $\begin{array}{l}\text { Lesion-pleural distance along the } \\
\text { needle path }(\mathrm{mm})^{3}\end{array}$ & $14.4(0.0-28.7)^{6}$ & $14.1(0.0-31.3)$ & $15.2(0.0-27.1)$ & 0.349 \\
\hline$=0.0$ & $75(34.6 \%)$ & $24(29.3 \%)$ & $51(37.8 \%)$ & \multirow{3}{*}{0.438} \\
\hline$>0.0$ and $<22.7$ & $70(32.3 \%)$ & $29(35.4 \%)$ & $41(30.4 \%)$ & \\
\hline$>22 . \overline{7}$ & $72(33.2 \%)$ & $29(35.4 \%)$ & $43(31.9 \%)$ & \\
\hline Needle-pleural angle $\left({ }^{\circ}\right)^{3}$ & $67.5(55.0-80.1)^{7}$ & $65.0(51.5-77.4)$ & $69.4(55.9-81.2)$ & 0.198 \\
\hline$\leq 67.5$ & $109(50.2 \%)$ & $45(54.9 \%)$ & $64(47.4 \%)$ & \multirow{2}{*}{0.286} \\
\hline$>67.5$ & $108(49.8 \%)$ & $37(45.1 \%)$ & $71(52.6 \%)$ & \\
\hline Number of obtained samples & $5(4-5)$ & $4(4-5)$ & $5(5-6)$ & $<0.001$ \\
\hline$\leq 5$ & $173(79.7 \%)$ & $80(97.6 \%)$ & $93(68.9 \%)$ & \multirow[b]{2}{*}{$<0.001$} \\
\hline$>5$ & $44(20.3 \%)$ & $2(2.4 \%)$ & $42(31.1 \%)$ & \\
\hline Operation time (min) & $18(14-25)$ & $25(21-31)$ & $15(12-20)$ & $<0.001$ \\
\hline Effective dose of X-ray (mSv) & $8.9(7.1-12.7)$ & $13.4(10.9-17.4)$ & $7.6(6.0-9.5)$ & $<0.001$ \\
\hline Complications & $57(26.3 \%)$ & $27(32.9 \%)$ & $30(22.2 \%)$ & 0.082 \\
\hline Pneumothorax & $31(14.3 \%)$ & $15(18.3 \%)$ & $16(11.9 \%)$ & 0.189 \\
\hline $\begin{array}{c}\text { Pulmonary } \\
\text { hemorrhage/hemoptysis }\end{array}$ & $47(21.7 \%)$ & $25(30.5 \%)$ & $22(16.3 \%)$ & 0.014 \\
\hline \multicolumn{5}{|l|}{ Severity grades of complication 8} \\
\hline Minor complications & $56(25.8 \%)$ & $26(31.7 \%)$ & $30(22.2 \%)$ & 0.121 \\
\hline$A$ & $25(11.5 \%)$ & $9(11.0 \%)$ & $16(11.9 \%)$ & \multirow{2}{*}{0.098} \\
\hline$B$ & $31(14.3 \%)$ & $17(20.7 \%)$ & $14(10.4 \%)$ & \\
\hline Major complications & $1(0.5 \%)$ & $1(1.2 \%)$ & $0(0.0 \%)$ & 0.121 \\
\hline C & $1(0.5 \%)$ & $1(1.2 \%)$ & $0(0.0 \%)$ & 0.098 \\
\hline
\end{tabular}


Table 2. Cont.

\begin{tabular}{|c|c|c|c|c|}
\hline & Total, $\mathrm{n}=217$ & $\begin{array}{l}\text { Conventional CT-Guided } \\
\text { Lung Biopsy, } \mathrm{n}=82 \text {. }\end{array}$ & $\begin{array}{c}\text { C-Arm CBCT Virtual } \\
\text { Navigation-Guided Lung } \\
\text { Biopsy, } n=135 \text {. }\end{array}$ & $p$-Value ${ }^{1}$ \\
\hline \multicolumn{5}{|l|}{ Pathological results of biopsies } \\
\hline Adenocarcinoma & $98(45.2 \%)$ & $38(46.3 \%)$ & $60(44.4 \%)$ & \multirow{10}{*}{0.714} \\
\hline Non-specific chronic inflammation & $34(15.7 \%)$ & $16(19.5 \%)$ & $18(13.3 \%)$ & \\
\hline Squamous cell carcinoma & $29(13.4 \%)$ & $11(13.4 \%)$ & $18(13.3 \%)$ & \\
\hline Tuberculosis & $15(6.9 \%)$ & $5(6.1 \%)$ & $10(7.4 \%)$ & \\
\hline Malignant cell type not specified & $11(5.1 \%)$ & $4(4.9 \%)$ & $7(5.2 \%)$ & \\
\hline Adenosquamous carcinoma & $10(4.6 \%)$ & $3(3.7 \%)$ & $7(5.2 \%)$ & \\
\hline Small cell carcinoma & $9(4.1 \%)$ & $3(3.7 \%)$ & $6(4.4 \%)$ & \\
\hline Organizing pneumonia & $5(2.3 \%)$ & $0(0.0 \%)$ & $5(3.7 \%)$ & \\
\hline Fungal infection & $4(1.8 \%)$ & $2(2.4 \%)$ & $2(1.5 \%)$ & \\
\hline Pneumoconiosis & $2(0.9 \%)$ & $0(0.0 \%)$ & $2(1.5 \%)$ & \\
\hline Accurate biopsy ${ }^{9}$ & $215(99.1 \%)^{10}$ & $81(98.8 \%)$ & $134(99.3 \%)$ & 1.000 \\
\hline
\end{tabular}

Note: Quantitative data were presented as median with inter-quantile range (IQR) and the counting data were presented as count with the percentage of the total in parenthesis. One or two cutpoints of quantitative variables were set up based on equal percentiles. ${ }^{1} p$-values comparing conventional CT- and C-arm CBCT virtual navigation-guided biopsies were determined using the Mann-Whitney test, Chi-square test, or Fisher's exact test. ${ }^{2}$ The lesion size was defined as an average of long- and short-axis measurements in the maximum lesion section of the axial CT image. ${ }^{3}$ Average of the measurements by two radiologists were involved in the statistical analysis. ${ }^{4}$ ICC was 0.974 (95\%CI: 0.964-0.981, $\left.p<0.001\right) .{ }^{5}$ ICC was $0.983(95 \%$ CI: $0.978-0.987, p<0.001) .{ }^{6}$ ICC was 0.973 (95\%CI: 0.965-0.980, $p<0.001$ ). ${ }^{7}$ ICC was 0.956 (95\%CI: $\left.0.943-0.966, p<0.001\right) .{ }^{8}$ The biopsy-related complications were classified under the updated standards of the Society of Interventional Radiology: $A$-No therapy, no sequences; $B$-Nominal therapy, no consequence, includes overnight admission for observation only; and $C$-Requires therapy, minor hospitalization $(<48 \mathrm{~h})$; in which minor complication was defined as Grade- $A$ or $-B$ complication, and major complication as Grade- $C$ complication. ${ }^{9}$ Accurate biopsy was defined as following surgical pathological diagnosis or subsequent clinical course for at least one year (e.g., growth or metastasis of malignant lesion, stable or regression of benign lesion) that were consistent with the biopsy pathological results. ${ }^{10}$ Two patients diagnosed with non-specific chronic inflammation by biopsies were finally confirmed with malignancies by surgical pathology (one was small cell carcinoma; the other was the metastasis of clear cell renal cell carcinoma).

\subsection{Risk Factor of Complications}

The rates of pneumothorax, pulmonary hemorrhage/hemoptysis, and overall complications were $14.3 \%$ (31/217), 21.7\% (47/217), and 26.3\% (57/217), respectively. The minor complications occupied $98.2 \%$ (56/57) of the overall complications (Table 2). Multiple factors were compared between patients with and without biopsy-related complications (incl. pneumothorax and pulmonary hemorrhage/hemoptysis) (Table 3). As a result, the incidence of biopsy-related complications was significantly higher in patients with intraprocedural needle repositioning than in those without repositioning ( $55.8 \%$ vs. $17.0 \%, p<0.001)$ (Table 3). Further univariate and multivariate logistic regression analyses revealed needle repositioning was the only independent risk factor of biopsy-related complications; the ORs of overall complications, pneumothorax, and pulmonary hemorrhage/hemoptysis were $6.169,10.463$, and 6.857, respectively ( $p<0.001$, each) (Table 4$)$. 
Table 3. Comparisons of factors between patients with and without biopsy-related complications.

\begin{tabular}{|c|c|c|c|c|c|c|c|c|c|}
\hline & \multicolumn{2}{|c|}{ Overall Complications } & \multirow[b]{2}{*}{$p$-Value ${ }^{1}$} & \multicolumn{2}{|c|}{ Pneumothorax } & \multirow[b]{2}{*}{$p$-Value $^{1}$} & \multicolumn{2}{|c|}{ Pulmonary Hemorrhage/Hemoptysis } & \multirow[b]{2}{*}{$p$-Value ${ }^{1}$} \\
\hline & $\begin{array}{c}\text { None, } \\
160 / 217(73.7 \%) \\
\end{array}$ & Yes, 57/217 (26.3\%) & & $\begin{array}{c}\text { None, } \\
186 / 217(85.7 \%) \\
\end{array}$ & Yes, 31/217 (14.3\%) & & $\begin{array}{c}\text { None, } \\
170 / 217(78.3 \%) \\
\end{array}$ & $\begin{array}{c}\text { Yes, } \\
n=47 / 217(21.7 \%)\end{array}$ & \\
\hline Age (years) & $62(53-69)$ & $64(55-69)$ & 0.175 & $62(53-69)$ & $64(61-67)$ & 0.075 & $62(53-68)$ & $64(55-71)$ & 0.096 \\
\hline$\leq 63$ & $89 / 115(77.4 \%)$ & $26 / 115(22.6 \%)$ & & $104 / 115(90.4 \%)$ & $11 / 115(9.6 \%)$ & & $94 / 115(81.7 \%)$ & $21 / 115(18.3 \%)$ & \\
\hline $\begin{array}{l}>63 \\
\text { Prior }\end{array}$ & $71 / 102(69.6 \%)$ & $31 / 102(30.4 \%)$ & 1.193 & $82 / 102(80.4 \%)$ & $20 / 102(19.6 \%)$ & 0.035 & $76 / 102(74.5 \%)$ & $26 / 102(25.5 \%)$ & 0.197 \\
\hline \multicolumn{10}{|l|}{$\begin{array}{c}\text { Prior } \\
\text { emphysema history }\end{array}$} \\
\hline $\begin{array}{l}\text { None } \\
\text { Yes }\end{array}$ & $\begin{array}{c}118 / 157(75.2 \%) \\
42 / 60(70.0 \%)\end{array}$ & $\begin{array}{c}39 / 157(24.8 \%) \\
18 / 60(30.0 \%)\end{array}$ & 0.440 & $\begin{array}{c}135 / 157(86.0 \%) \\
51 / 60(85.0 \%)\end{array}$ & $\begin{array}{c}22 / 157(14.0 \%) \\
9 / 60(15.0 \%)\end{array}$ & 0.853 & $\begin{array}{c}124 / 157(79.0 \%) \\
46 / 60(76.7 \%)\end{array}$ & $\begin{array}{c}33 / 157(21.0 \%) \\
14 / 60(23.3 \%)\end{array}$ & 0.711 \\
\hline Guidance & & & & & & & & & \\
\hline Conventional CT & $55 / 82(67.1 \%)$ & $27 / 82(32.9 \%)$ & 0.082 & $67 / 82(81.7 \%)$ & $15 / 82(18.3 \%)$ & 0.189 & $57 / 82(69.5 \%)$ & $25 / 82(30.5 \%)$ & \\
\hline $\begin{array}{c}\text { CBCT } \\
\text { Lesion size }(\mathrm{mm})^{2,3}\end{array}$ & $\begin{array}{l}105 / 135(77.8 \%) \\
39.9(27.3-54.5)\end{array}$ & $\begin{array}{l}30 / 135(22.2 \%) \\
40.0(26.5-54.8)\end{array}$ & $\begin{array}{l}0.082 \\
0.755\end{array}$ & $\begin{array}{l}119 / 135(88.1 \%) \\
40.1(26.8-54.8)\end{array}$ & $\begin{array}{l}16 / 135(11.9 \%) \\
37.0(24.5-55.3)\end{array}$ & $\begin{array}{l}0.189 \\
0.696\end{array}$ & $\begin{array}{c}113 / 135(83.7 \%) \\
40.1(27.8-55.3)\end{array}$ & $\begin{array}{l}22 / 135(16.3 \%) \\
39.0(24.5-53.8)\end{array}$ & $\begin{array}{l}0.014 \\
0.462\end{array}$ \\
\hline$\leq 40.0$ & $81 / 110(73.6 \%)$ & $29 / 110(26.4 \%)$ & & $93 / 110(84.5 \%)$ & $17 / 110(15.5 \%)$ & & $85 / 110(77.3 \%)$ & $25 / 110(22.7 \%)$ & \\
\hline$>40.0$ & $79 / 107(73.8 \%)$ & $28 / 107(26.2 \%)$ & 0.974 & $93 / 107(86.9 \%)$ & $14 / 107(13.1 \%)$ & 0.618 & $85 / 107(79.4 \%)$ & $22 / 107(20.6 \%)$ & 0.698 \\
\hline \multicolumn{10}{|l|}{ Position } \\
\hline Supine & $85 / 113(75.2 \%)$ & $28 / 113(24.8 \%)$ & & $100 / 113(88.5 \%)$ & $13 / 113(11.5 \%)$ & & $89 / 113(78.8 \%)$ & $24 / 113(21.2 \%)$ & \\
\hline Prone & $71 / 99(71.7 \%)$ & $28 / 99(28.3 \%)$ & 0.803 & $82 / 99(82.8 \%)$ & $17 / 99(17.2 \%)$ & 0.468 & $77 / 99(77.8 \%)$ & $22 / 99(22.2 \%)$ & 0.981 \\
\hline Lateral recumbent & $4 / 5(80.0 \%)$ & $1 / 5(20.0 \%)$ & & $4 / 5(80.0 \%)$ & $1 / 5(20.0 \%)$ & & $4 / 5(80.0 \%)$ & $1 / 5(20.0 \%)$ & \\
\hline $\begin{array}{c}\text { Number of } \\
\text { needle repositioning }\end{array}$ & $0(0-0)$ & $1(0-1)$ & $<0.001$ & $0(0-0)$ & $1(0-2)$ & $<0.001$ & $0(0-0)$ & $1(0-2)$ & $<0.001$ \\
\hline \multicolumn{10}{|l|}{ Needle repositioning } \\
\hline $\begin{array}{l}\text { None } \\
\text { Yes }\end{array}$ & $\begin{array}{c}137 / 165(83.0 \%) \\
23 / 52(44.2 \%)\end{array}$ & $\begin{array}{l}28 / 165(17.0 \%) \\
29 / 52(55.8 \%)\end{array}$ & $<0.001$ & $\begin{array}{c}154 / 165(93.3 \%) \\
32 / 52(61.5 \%)\end{array}$ & $\begin{array}{l}11 / 165(6.7 \%) \\
20 / 52(38.5 \%)\end{array}$ & $<0.001$ & $\begin{array}{c}144 / 165(87.3 \%) \\
26 / 52(50.0 \%)\end{array}$ & $\begin{array}{l}21 / 165(12.7 \%) \\
26 / 52(50.0 \%)\end{array}$ & $<0.001$ \\
\hline \multicolumn{10}{|l|}{$\begin{array}{l}\text { Lesion-skin distance } \\
\text { along the needle }\end{array}$} \\
\hline $\begin{array}{l}\leq 51.7 \\
>51.7\end{array}$ & $84 / 108(77.8 \%)$ & $24 / 108(22.2 \%)$ & 0.178 & $97 / 108(89.8 \%)$ & $11 / 108(10.2 \%)$ & 0.086 & $86 / 108(79.6 \%)$ & $22 / 108(20.4 \%)$ & 0.646 \\
\hline \multirow{2}{*}{\multicolumn{10}{|c|}{$\begin{array}{l}\text { Lesion-pleural } \\
\text { distance along the }\end{array}$}} \\
\hline & $13.6(0.0-27.3)$ & $16.3(0.0-32.4)$ & 0.228 & $13.1(0.0-27.5)$ & $19.5(6.4-38.2)$ & 0.046 & $14.3(0.0-28.1)$ & $14.5(0.0-31.1)$ & 0.550 \\
\hline & $60 / 75(80.0 \%)$ & $15 / 75(20.0 \%)$ & & $69 / 75(92.0 \%)$ & $6 / 75(8.0 \%)$ & & $62 / 75(82.7 \%)$ & $13 / 75(17.3 \%)$ & \\
\hline $\begin{array}{l}>0.0 \text { and } \leq 22.7 \\
>22.7\end{array}$ & $48 / 70(68.6 \%)$ & $22 / 70(31.4 \%)$ & 0.277 & $57 / 70(81.4 \%)$ & $13 / 70(18.6 \%)$ & 0.149 & $51 / 70(72.9 \%)$ & $19 / 70(27.1 \%)$ & 0.351 \\
\hline \multirow{3}{*}{$\begin{array}{c}>22.7 \\
\text { Needle-pleural } \\
\text { angle }\left(^{\circ}\right)^{3} \\
\leq 67.5 \\
>67.5\end{array}$} & $52 / 72(72.2 \%)$ & 20/72 (27.8\%) & & $60 / 72(83.3 \%)$ & 12/72 (16.7\%) & & $57 / 72(79.2 \%)$ & $15 / 72(20.8 \%)$ & \\
\hline & $68.7(55.5-80.7)$ & $65.0(50.3-77.2)$ & 0.298 & $67.9(55.0-80.2)$ & $63.4(51.0-79.1)$ & 0.576 & $68.7(55.9-80.4)$ & $63.7(50.3-77.0)$ & 0.262 \\
\hline & $\begin{array}{l}76 / 109(69.7 \%) \\
84 / 108(77.8 \%)\end{array}$ & $\begin{array}{l}33 / 109(30.3 \%) \\
24 / 108(22.2 \%)\end{array}$ & 0.178 & $\begin{array}{l}92 / 109(84.4 \%) \\
94 / 108(87.0 \%)\end{array}$ & $\begin{array}{l}17 / 109(15.6 \%) \\
14 / 108(13.0 \%)\end{array}$ & 0.579 & $\begin{array}{l}81 / 109(74.3 \%) \\
89 / 108(82.4 \%)\end{array}$ & $\begin{array}{l}28 / 109(25.7 \%) \\
19 / 108(17.6 \%)\end{array}$ & 0.148 \\
\hline \multirow{2}{*}{$\begin{array}{c}\begin{array}{c}\text { Number of } \\
\text { obtained samples } \\
\leq 5 \\
>5\end{array}\end{array}$} & $5(4-5)$ & $5(4-5)$ & 0.605 & $5(4-5)$ & $5(4-5)$ & 0.616 & $5(4-5)$ & $5(4-5)$ & 0.287 \\
\hline & $\begin{array}{c}126 / 173(72.8 \%) \\
34 / 44(77.3 \%)\end{array}$ & $\begin{array}{l}47 / 173(27.2 \%) \\
10 / 44(22.7 \%)\end{array}$ & 0.550 & $\begin{array}{c}148 / 173(85.5 \%) \\
38 / 44(86.4 \%)\end{array}$ & $\begin{array}{c}25 / 173(14.5 \%) \\
6 / 44(13.6 \%)\end{array}$ & 0.890 & $\begin{array}{c}132 / 173(76.3 \%) \\
38 / 44(86.4 \%)\end{array}$ & $\begin{array}{c}41 / 173(23.7 \%) \\
6 / 44(13.6 \%)\end{array}$ & 0.148 \\
\hline
\end{tabular}


Table 4. Univariate and multivariate logistic regression analysis.

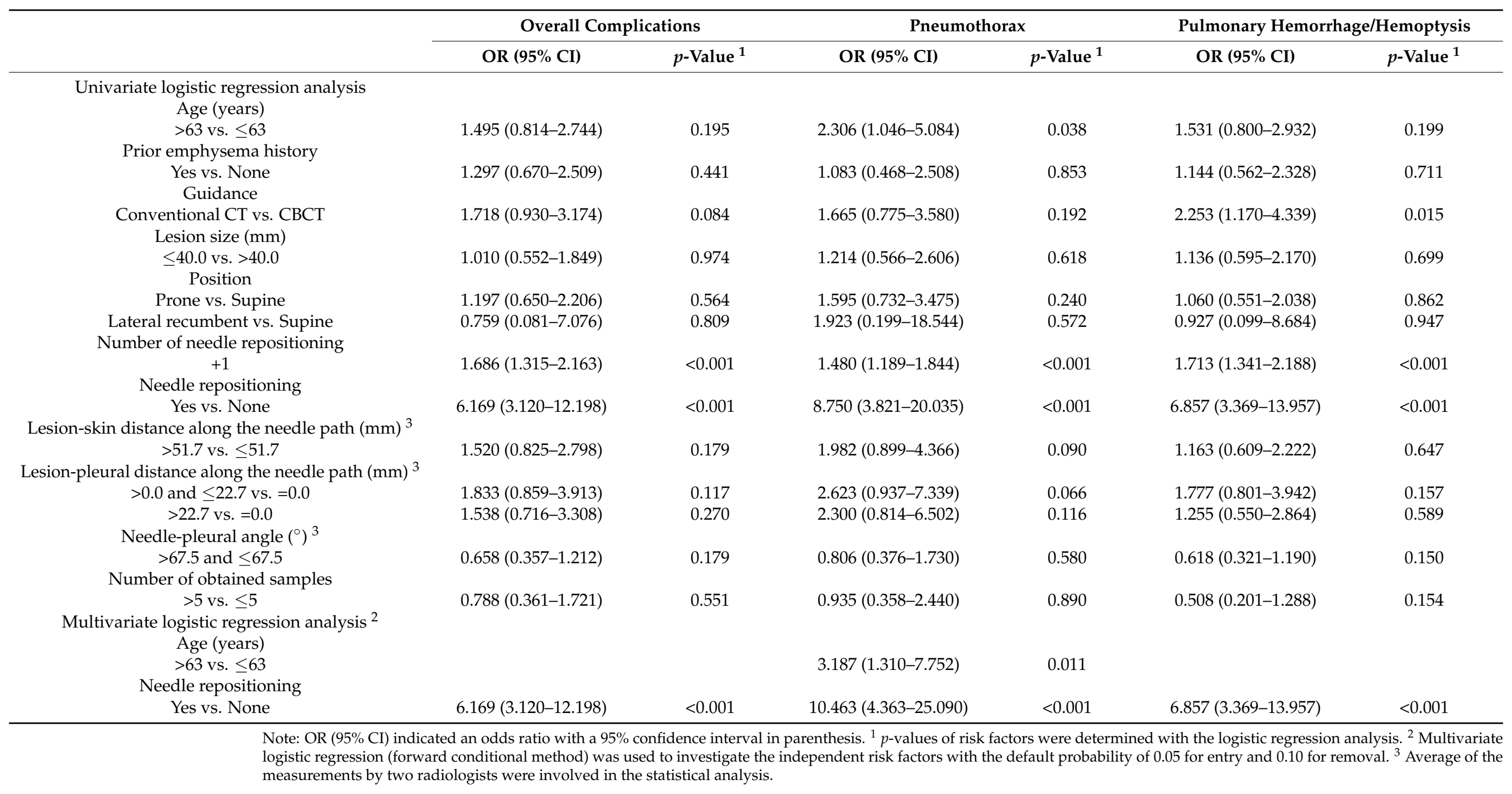




\section{Discussion}

Our retrospective study finds that the C-arm CBCT virtual navigation with real-time fluoroscopy monitoring in lung biopsy could reduce the incidence of needle repositioning compared to conventional CT guidance $(6.7 \%$ vs. $52.4 \%, p<0.001)$. It shortened the operation time and lowered the effective dose of X-ray (15 min vs. $25 \mathrm{~min}$ and $7.6 \mathrm{mSv}$ vs. $13.4 \mathrm{mSv}$, respectively; $p<0.001$, both). The technical success rates were $100 \%$ in both biopsies, with biopsy accuracies of over $98 \%$. The overall complication incidence was $26.3 \%(57 / 217)$, most of which are minor complications $(56 / 57,98.2 \%)$. Further logistic regression analysis revealed that needle repositioning during the procedure was the only independent risk factor for biopsy-related complications (OR: 6.169, $p<0.001$ ). Our results suggest that needle repositioning increases the risk of pneumothorax and pulmonary hemorrhage/hemoptysis by approximately 10 and 7 times, respectively.

In a previous study involving a total of 58 patients, $C$-arm CBCT virtual navigation presented more favorable merits than conventional CT guidance in biopsies at different organs [6]. It brought about less needle repositioning, similar to the results in lung biopsies of our study [6]. However, the number of needle repositioning and operation time in our conventional CT-guidance group was significantly lower and shorter than in the previous report (One vs. 2 and $25 \mathrm{~min}$ vs. $>30 \mathrm{~min}$, respectively), indicating the importance of proficient skills for biopsies under conventional CT guidance [6]. Even so, C-arm CBCT virtual navigation in our study still significantly reduced the needle repositioning incidence compared with conventional CT guidance $(6.7 \%$ vs. $52.4 \%, p<0.001)$. These findings suggest $\mathrm{C}$-arm $\mathrm{CBCT}$ virtual navigation is better than conventional $\mathrm{CT}$ guidance in coaxial needle positioning. Although direct comparison between the effective dose of conventional $\mathrm{CT}$ and $\mathrm{CBCT}$ is impossible, standard algorithmic conversion was established from previous studies $[10,14,21,22]$. As a result, a median effective dose was $7.6 \mathrm{mSv}$ under C-arm CBCT virtual navigation with a $43.3 \%$ reduction compared with conventional $C T$, similar to previous reports $[6,10,12,14,25,26]$. As an alternative, fluoroscopic CT guidance can also monitor the needle positioning in real-time [25-28]. However, fluoroscopic CT delivers higher radiation doses to both the patient and operator than conventional $\mathrm{CT}$, much more than CBCT $[18,29]$. So, we do not prefer it in our center.

Although controlled studies are few so far, by comparing with other research, it was reported that the biopsy accuracy was higher in C-arm CBCT virtual navigation than conventional CT guidance (98.2\% vs. $83.7 \%$ ) when diagnosing small lung solid lesions less than 15-20 mm $[4,10]$. However, our study did not find a significant difference in the biopsy accuracy between these two techniques (>98\%, both), similar to one previous study [26]. A larger lesion size (median: $40 \mathrm{~mm}$ ) with a shorter lesion-pleural distance (median: $14 \mathrm{~mm}$ ) in our cohort probably accounted for this discrepancy from previous studies, because it was reported that the benefit of increased biopsy accuracy under C-arm CBCT virtual navigation was gradually demolished with the increase of the lesion size [4,9-11]. After all, the small lesion size is a risk factor of technical failure and false-negative results in either CT- or CBCT-guided lung biopsy $[4,10]$.

In accordance with previous studies, we identified pneumothorax, pulmonary hemorrhage, and hemoptysis were the most common biopsy-related complications with an average incidence of about 25\% [2,3,7,10-12,14,20,30]. A major complication rate of only $2.4-3.8 \%$ was reported, indicating most complications did not require additional treatment $[7,10]$. However, there was a significant variation of the reported complication incidence, such as the pneumothorax rate of $0-61 \%$ and the pulmonary hemorrhage/hemoptysis rate of $0-41 \%$ [2,3,7,10-12,14,20,30]. Nevertheless, unlike published studies, pulmonary hemorrhage/hemoptysis had a higher incidence than pneumothorax (21.7\% vs. $14.3 \%)$ in our cohort $[10,12,14,20]$. It probably ascribes to: 1 . more samples were obtained in our cohort than in previous studies (5 vs. 2-3); and 2. blebs or bullae were avoided in the puncture process $[10,12,14,20]$. Additionally, we did not observe other complications, including air embolism, hemothorax, and tumoral seeding [2]. 
Our study revealed that needle repositioning significantly increased the risk of pneumothorax and pulmonary hemorrhage/hemoptysis, likely because it resulted in multiple transverses of the lung parenchyma and pleura [6]. Thus, reducing needle manipulations is essential in a biopsy, no matter which guidance is used. Furthermore, extended lesionpleura distance was also documented to be significantly associated with the increased risks of pneumothorax and pulmonary hemorrhage/hemoptysis $[2,4,14,31]$. However, our study does not support this finding, probably because bronchoscopic biopsy is more recommended to obtain samples for central lung lesions, resulting in the majority of the peripheral lung lesions in our cohort [2]. It can also explain why rare major complication $(1 / 217,0.5 \%)$ was observed in our cohort. In addition, some other risk factors were once reported. For instance, ground-glass nodule (GGN) was associated with pulmonary hemorrhage/hemoptysis and emphysema with pneumothorax $[2,10,14,20]$. However, GGN was not biopsied in our center following the guidelines [3]. Moreover, to reduce the biopsyrelated complications, methods such as positioning a biopsy side down and puncture under an acute needle-pleural angle $\left(<51^{\circ}\right)$ were suggested $[20,23]$. However, our data did not support these findings because a rare lateral recumbent position and larger needle-pleural angle (median: $67.5^{\circ}$ ) were applied.

Our study has several limitations. First, it was retrospectively carried out in a single center, and all patients underwent biopsies by the same interventionalists team. However, our results indicate that even if the operators are more skillful in conventional-CT guided biopsy, C-arm CBCT virtual navigation can still facilitate the accurate needle puncture, reducing the need for needle repositioning. Second, small or central lung lesions were rarely involved in our cohort, bringing about some discrepancies from previous studies $[4,10]$. In these cases, surgical resection or bronchoscopic biopsy was more recommended following guidelines or recommendations [2,3,32]. Third, histologic confirmation from surgical resection did not apply to all patients, similar to most previous studies $[6,7,10,23]$. In this case, a long-term follow-up (at least one year) was necessary to identify the malignant or benign diagnosis $[6,7,10,23]$.

\section{Conclusions}

This retrospective case-control study demonstrates that both the conventional CT- and $\mathrm{C}$-arm $\mathrm{CBCT}$ virtual navigation-guided lung biopsies are safe and accurate. However, the $\mathrm{C}$-arm $\mathrm{CBCT}$ virtual navigation facilitates the needle positioning and reduces the radiation dose, compared to conventional CT guidance. Since needle repositioning significantly increased the incidence of biopsy-related complications, as revealed in our study, C-arm $\mathrm{CBCT}$ virtual navigation can be more suggested in transthoracic lung biopsy to reduce the necessity of needle repositioning and the subsequent complications.

Author Contributions: F.P., H.L. and C.Z. contributed to the conception and design of the study. L.L., D.L., X.W. and W.Z. performed the acquisition of the data. L.Y. and Y.W. performed the CT measurement work. F.P., L.L. and D.L. interpreted the data. F.P., L.Y. and Y.W. wrote sections of the manuscript. All authors have read and agreed to the published version of the manuscript.

Funding: This study was supported by the National Natural Science Foundation of China [82172034].

Institutional Review Board Statement: The study was conducted according to the guidelines of the Declaration of Helsinki and approved by the Ethics Committee of Union Hospital, Tongji Medical College, Huazhong University of Science and Technology (protocol code: 2021-IEC237; date of approval: 3 March 2021).

Informed Consent Statement: The informed consent of patients was waived by the Ethics Committee of Union Hospital, Tongji Medical College, Huazhong University of Science and Technology due to the retrospective analysis of the privacy-preserving anonymous data.

Data Availability Statement: The anonymous data presented in this study are available on request from the corresponding author. The data are not publicly available due to institutional regulations. 
Acknowledgments: We are grateful to Ke Jiang (Department of Thoracic Surgery, Union Hospital, Tongji Medical College, Huazhong University of Science and Technology,430022 Wuhan) and Jiazheng Wang (MSC Clinical \& Technical Solutions, Philips Healthcare, Beijing, 100000, China) for many valuable suggestions through the conception and design of this work. Also, we would like to express our gratitude to Yuyang Chen (Putnam Science Academy, CT 06260, USA) for language editing.

Conflicts of Interest: The authors declare no conflict of interest.

$\begin{array}{ll}\text { Abbreviations } \\ \text { CBCT } & \text { cone-beam computed tomography } \\ \text { DLP } & \text { dose-linear product } \\ \text { DAP } & \text { dose-area product } \\ \text { GGN } & \text { ground-glass nod0ule } \\ \text { ICC } & \text { intraclass correlation efficient } \\ \text { IQR } & \text { inter-quantile range } \\ \text { OR } & \text { odds ratio } \\ 95 \% \text { CI } & 95 \% \text { confidence interval }\end{array}$

\section{References}

1. Manhire, A.R.; Richardson, C.M.; Gleeson, F.V. Lung biopsy guidelines-for the obedience of fools and guidance of wise men. Thorax 2003, 58, 913-914. [CrossRef] [PubMed]

2. de Margerie-Mellon, C.; de Bazelaire, C.; de Kerviler, E. Image-guided biopsy in primary lung cancer: Why, when and how. Diagn. Interv. Imaging 2016, 97, 965-972. [CrossRef]

3. Manhire, A.; Charig, M.; Clelland, C.; Gleeson, F.; Miller, R.; Moss, H.; Pointon, K.; Richardson, C.; Sawicka, E. Guidelines for radiologically guided lung biopsy. Thorax 2003, 58, 920-936. [CrossRef] [PubMed]

4. Huang, M.D.; Weng, H.H.; Hsu, S.L.; Hsu, L.S.; Lin, W.M.; Chen, C.W.; Tsai, Y.H. Accuracy and complications of CT-guided pulmonary core biopsy in small nodules: A single-center experience. Cancer Imaging 2019, 19, 51. [CrossRef]

5. Jin, K.N.; Park, C.M.; Goo, J.M.; Lee, H.J.; Lee, Y.; Kim, J.I.; Choi, S.Y.; Kim, H.C. Initial experience of percutaneous transthoracic needle biopsy of lung nodules using C-arm cone-beam CT systems. Eur. Radiol. 2010, 20, 2108-2115. [CrossRef]

6. Abi-Jaoudeh, N.; Fisher, T.; Jacobus, J.; Skopec, M.; Radaelli, A.; Van Der Bom, I.M.; Wesley, R.; Wood, B.J. Prospective Randomized Trial for Image-Guided Biopsy Using Cone-Beam CT Navigation Compared with Conventional CT. J. Vasc. Interv. Radiol. 2016, 27, 1342-1349. [CrossRef]

7. Braak, S.J.; Herder, G.J.; van Heesewijk, J.P.; van Strijen, M.J. Pulmonary masses: Initial results of cone-beam CT guidance with needle planning software for percutaneous lung biopsy. Cardiovasc. Interv. Radiol. 2012, 35, 1414-1421. [CrossRef]

8. Hwang, H.S.; Chung, M.J.; Lee, J.W.; Shin, S.W.; Lee, K.S. C-arm cone-beam CT-guided percutaneous transthoracic lung biopsy: Usefulness in evaluation of small pulmonary nodules. AJR Am. J. Roentgenol. 2010, 195, W400-W407. [CrossRef]

9. Cheung, J.Y.; Kim, Y.; Shim, S.S.; Lim, S.M. Combined fluoroscopy- and CT-guided transthoracic needle biopsy using a C-arm cone-beam CT system: Comparison with fluoroscopy-guided biopsy. Korean J. Radiol. 2011, 12, 89-96. [CrossRef]

10. Choi, J.W.; Park, C.M.; Goo, J.M.; Park, Y.K.; Sung, W.; Lee, H.J.; Lee, S.M.; Ko, J.Y.; Shim, M.S. C-arm cone-beam CT-guided percutaneous transthoracic needle biopsy of small $(\leq 20 \mathrm{~mm})$ lung nodules: Diagnostic accuracy and complications in 161 patients. AJR Am. J. Roentgenol. 2012, 199, W322-W330. [CrossRef]

11. Choi, M.J.; Kim, Y.; Hong, Y.S.; Shim, S.S.; Lim, S.M.; Lee, J.K. Transthoracic needle biopsy using a C-arm cone-beam CT system: Diagnostic accuracy and safety. Br. J. Radiol. 2012, 85, e182-e187. [CrossRef]

12. Choo, J.Y.; Park, C.M.; Lee, N.K.; Lee, S.M.; Lee, H.J.; Goo, J.M. Percutaneous transthoracic needle biopsy of small ( $\leq 1 \mathrm{~cm})$ lung nodules under C-arm cone-beam CT virtual navigation guidance. Eur. Radiol. 2013, 23, 712-719. [CrossRef]

13. Hwang, E.J.; Kim, H.; Park, C.M.; Yoon, S.H.; Lim, H.J.; Goo, J.M. Cone beam computed tomography virtual navigation-guided transthoracic biopsy of small $(\leq 1 \mathrm{~cm})$ pulmonary nodules: Impact of nodule visibility during real-time fluoroscopy. Br. J. Radiol. 2018, 91, 20170805. [CrossRef]

14. Lee, S.M.; Park, C.M.; Lee, K.H.; Bahn, Y.E.; Kim, J.I.; Goo, J.M. C-arm cone-beam CT-guided percutaneous transthoracic needle biopsy of lung nodules: Clinical experience in 1108 patients. Radiology 2014, 271, 291-300. [CrossRef]

15. Jiao, D.; Yuan, H.; Zhang, Q.; Han, X. Flat detector C-arm CT-guided transthoracic needle biopsy of small $(\leq 2.0 \mathrm{~cm})$ pulmonary nodules: Diagnostic accuracy and complication in 100 patients. Radiol. Med. 2016, 121, 268-278. [CrossRef] [PubMed]

16. Jiao, D.C.; Li, Z.M.; Yuan, H.F.; Zhang, Q.H.; Ren, J.Z.; Zhou, P.L.; Wu, G.; Han, X.W. Flat detector C-arm CT-guidance system in performing percutaneous transthoracic needle biopsy of small $(\leq 3 \mathrm{~cm})$ pulmonary lesions. Acta Radiol. 2016, 57, 677-683. [CrossRef] [PubMed]

17. Hwang, E.J.; Park, C.M.; Yoon, S.H.; Lim, H.J.; Goo, J.M. Risk factors for haemoptysis after percutaneous transthoracic needle biopsies in 4172 cases: Focusing on the effects of enlarged main pulmonary artery diameter. Eur. Radiol. 2018, 28, 1410-1419. [CrossRef] [PubMed] 
18. Winokur, R.S.; Pua, B.B.; Sullivan, B.W.; Madoff, D.C. Percutaneous lung biopsy: Technique, efficacy, and complications. Semin. Interv. Radiol. 2013, 30, 121-127. [CrossRef] [PubMed]

19. Bankier, A.A.; MacMahon, H.; Goo, J.M.; Rubin, G.D.; Schaefer-Prokop, C.M.; Naidich, D.P. Recommendations for Measuring Pulmonary Nodules at CT: A Statement from the Fleischner Society. Radiology 2017, 285, 584-600. [CrossRef]

20. Drumm, O.; Joyce, E.A.; de Blacam, C.; Gleeson, T.; Kavanagh, J.; McCarthy, E.; McDermott, R.; Beddy, P. CT-guided Lung Biopsy: Effect of Biopsy-side Down Position on Pneumothorax and Chest Tube Placement. Radiology 2019, 292, 190-196. [CrossRef]

21. McCollough, C.; Edyvean, S.; Gould, B.; Keat, N.; Judy, P.; Kalender, W.; Morin, R.; Payne, T.; Stern, S.; Rothenberg, L. The Measurement, Reporting, and Management of Radiation Dose in CT. AAPM Rep. 2008, 96, 11-13.

22. Deak, P.D.; Smal, Y.; Kalender, W.A. Multisection CT protocols: Sex- and age-specific conversion factors used to determine effective dose from dose-length product. Radiology 2010, 257, 158-166. [CrossRef]

23. Li, Y.; Du, Y.; Yang, H.F.; Yu, J.H.; Xu, X.X. CT-guided percutaneous core needle biopsy for small ( $\leq 20 \mathrm{~mm})$ pulmonary lesions. Clin. Radiol. 2013, 68, e43-e48. [CrossRef]

24. Khalilzadeh, O.; Baerlocher, M.O.; Shyn, P.B.; Connolly, B.L.; Devane, A.M.; Morris, C.S.; Cohen, A.M.; Midia, M.; Thornton, R.H.; Gross, K.; et al. Proposal of a New Adverse Event Classification by the Society of Interventional Radiology Standards of Practice Committee. J. Vasc. Interv. Radiol. 2017, 28, 1432-1437.e3. [CrossRef]

25. Fior, D.; Vacirca, F.; Leni, D.; Pagni, F.; Ippolito, D.; Riva, L.; Sironi, S.; Corso, R. Virtual Guidance of Percutaneous Transthoracic Needle Biopsy with C-Arm Cone-Beam CT: Diagnostic Accuracy, Risk Factors and Effective Radiation Dose. Cardiovasc. Interv. Radiol. 2019, 42, 712-719. [CrossRef] [PubMed]

26. Cheng, Y.C.; Tsai, S.H.; Cheng, Y.; Chen, J.H.; Chai, J.W.; Chen, C.C. Percutaneous Transthoracic Lung Biopsy: Comparison Between C-Arm Cone-Beam CT and Conventional CT Guidance. Transl. Oncol. 2015, 8, 258-264. [CrossRef] [PubMed]

27. Jiao de, C.; Li, T.F.; Han, X.W.; Wu, G.; Ma, J.; Fu, M.T.; Sun, Q.; Beilner, J. Clinical applications of the C-arm cone-beam CT-based 3D needle guidance system in performing percutaneous transthoracic needle biopsy of pulmonary lesions. Diagn Interv. Radiol. 2014, 20, 470-474. [CrossRef]

28. Floridi, C.; Muollo, A.; Fontana, F.; Rotolo, N.; Ierardi, A.M.; Duka, E.; Pellegrino, C.; Carrafiello, G. C-arm cone-beam computed tomography needle path overlay for percutaneous biopsy of pulmonary nodules. Radiol. Med. 2014, 119, 820-827. [CrossRef] [PubMed]

29. Strocchi, S.; Colli, V.; Conte, L. Multidetector CT fluoroscopy and cone-beam CT-guided percutaneous transthoracic biopsy: Comparison based on patient doses. Radiat. Prot. Dosim. 2012, 151, 162-165. [CrossRef]

30. Gulias-Soidan, D.; Crus-Sanchez, N.M.; Fraga-Manteiga, D.; Cao-Gonzalez, J.I.; Balboa-Barreiro, V.; Gonzalez-Martin, C. ConeBeam CT-Guided Lung Biopsies: Results in 94 Patients. Diagnostics 2020, 10, 1068. [CrossRef] [PubMed]

31. Wang, Y.; Li, W.; He, X.; Li, G.; Xu, L. Computed tomography-guided core needle biopsy of lung lesions: Diagnostic yield and correlation between factors and complications. Oncol. Lett. 2014, 7, 288-294. [CrossRef] [PubMed]

32. Ettinger, D.S.; Wood, D.E.; Aisner, D.L.; Akerley, W.; Bauman, J.R.; Bharat, A.; Bruno, D.S.; Chang, J.Y.; Chirieac, L.R.; D'Amico, T.A.; et al. NCCN Guidelines Insights: Non-Small Cell Lung Cancer, Version 2.2021. J. Natl. Compr. Cancer Netw. 2021, 19, 254-266. [CrossRef] [PubMed] 